\title{
Estrés oxidativo e inflamación en insuficiencia cardiaca: Mecanismos de daño y alternativas terapéuticas
}

\author{
Rodrigo Miranda $\mathrm{H}^{1}$, Pablo Castro $\mathrm{G}^{1}$, Hugo Verdejo $\mathrm{P}^{1}$, \\ Mario Chiong2, G uillermo Díaz-Araya², Rosemarie Mellado², \\ Diego Rojas², Roberto Concepción ${ }^{3}$, Sergio Lavandero². \\ Oxidative stress and inflammation \\ in heart failure: Mechanisms \\ of damage and therapeutic alternatives
}

Despite advances in treatment, chronic heart failure still is associated with a poor prognosis and remains a leading cause of cardiovascular death. Cumulating evidence suggests that imbalances in redox state lead to a higher generation of reactive oxygen species. This phenomenon, along with pro-inflammatory cytokine activation and extra cellular matrix alterations with reactive fibrosis, play an important role in the pathogenesis and progression of heart failure, through the development of endothelial and myocardial dysfunction. The understanding of the underlying phenomena and the metabolic pathways involved will allow further development of therapies aiming to change the natural history of heart failure (Rev Méd Chile 2007; 135: 1056-63).

(Key words: Endothelial cells; Heart failure, congestive; Oxidative stress)

\begin{abstract}
Recibido el 3 de julio, 2006. Aceptado el 6 de noviembre, 2006.
Trabajo financiado por Fondecyt \#1050768.

${ }^{1}$ Facultad de Medicina, Pontificia Universidad Católica de Chile. ${ }^{2}$ Centro FONDAP de Estudios Moleculares, Facultad de Ciencias Químicas y Farmacéuticas, Universidad de Chile. ${ }^{3}$ Hospital Dipreca. Santiago de Chile.
\end{abstract}

P ese a los avances en diagnóstico y terapia, la insuficiencia cardiaca (IC) sigue siendo una de las causas más importantes de hospitalización, morbilidad y mortalidad a nivel mundial ${ }^{1}$.

En la actualidad entendemos que la IC se produce no sólo como consecuencia de un proce-

Correspondencia a: Pablo Castro. Pontificia Universidad Católica de Chile. Fono: 56-2-3543624. E mail: pcastro@med.puc.cl Sergio Lavandero. Universidad de Chile. Fax: 56-2-6392037. E mail: slavander@uchile.cl so hemodinámico de retención de agua y sal, sino como un síndrome complejo con importantes alteraciones neurohumorales que contribuyen a los síntomas y progresión de la enfermedad ${ }^{1}$.

La búsqueda de terapias efectivas para IC, ha impulsado la investigación de los mecanismos fisiopatológicos subyacentes. Actualmente, la IC es vista como un fenómeno complejo resultante de la interacción de alteraciones locales a nivel del cardiomiocito (por ejemplo, trastornos en la homeostasis de calcio o de la activación de proteínas contráctiles) y alteraciones sistémicas resultantes 
de respuestas compensadoras desadaptativas (activación simpática y neurohumoral) o secundarias a la incapacidad del miocardio de suplir las demandas metabólicas de los tejidos periféricos (estrés oxidativo e inflamación). Dichas alteraciones se traducen en cambios morfológicos y funcionales a nivel de miocardio (hipertrofia, fibrosis, inflamación y apoptosis), referidos habitualmente como remodelado ventricular, y en la totalidad del árbol vascular con aumento del tono vasomotor y disfunción endotelial ${ }^{2}$.

Sabemos que las diversas noxas que afectan el corazón, producen disminución de la eficacia contráctil y aumento del estrés oxidativo con disminución de sistemas de defensa antioxidantes. Hay activación neurohumoral y aumento de citoquinas inflamatorias. Estos fenómenos contribuyen en la expresión clínica y progresión de la falla cardiaca (Figura 1).

La interacción entre los mecanismos citados no está bien definida. Estudios recientes han mostrado un aumento en los marcadores de estrés oxidativo en IC, secundario a una deficiente utilización del oxígeno a nivel periférico por desacoplamiento de la cadena respiratoria, que se traduce en la generación de productos tóxicos conocidos como especies reactivas del oxígeno $(\mathrm{ROS})^{3}$. Si esto es un evento primario en la fisiopatología de la IC, o secundario a procesos de isquemia-reperfusión 0 a la activación neurohumoral, es aún materia de debate.

Durante los últimos años, nuestro grupo ha desarrollado una línea de investigación dirigida al estudio del estrés oxidativo y la inflamación en IC y su relación con cambios en la vasculatura periférica (disfunción endotelial), evaluando el posible beneficio clínico de intervenciones terapéuticas sobre estos procesos.

El objetivo de esta revisión es la de evaluar la evidencia actual sobre el rol del estrés oxidativo y la inflamación en IC y los potenciales alcances terapéuticos de este conocimiento.

\section{ESTRÉS OXIDATIVO FN LA INSUFICIENCIA CARDIACA}

El rol de las ROS en la patogenia y progresión de la IC ha adquirido considerable relevancia en los

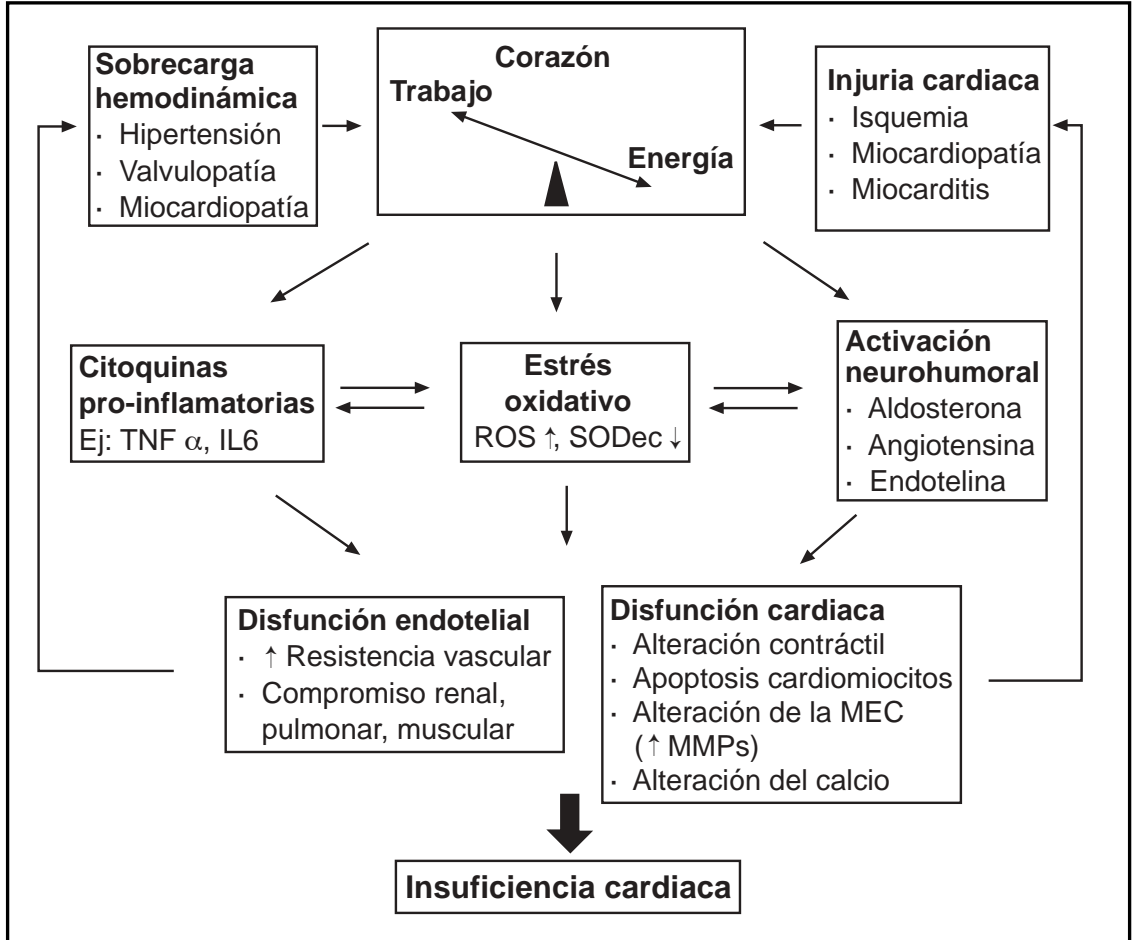

Figura 1. Progresión de la falla cardiaca y rol del estrés oxidativo. Diversas noxas afectan el corazón causando estrés oxidativo, con aumento de especies reactivas del oxígeno (ROS) y disminución de sistemas de defensa antioxidantes como superóxido dismutasa (SODec). Hay activación neurohumoral y aumento de citoquinas como factor de necrosis tumoral $\alpha$ (TNFa) e interleuquina 6 (IL-6). Estas alteraciones producen disfunción endotelial y cardiaca, con alteración de la matriz extracelular (MEC) y aumento de metaloproteinasas (MMPs). 
últimos años. En un artículo reciente, nuestro grupo demostró la existencia de un estado de estrés redox aumentado en pacientes con IC avanzada, evidenciado por elevación del malondialdehido plasmático (MDA), un producto de peroxidación lipídica, junto a la depleción de la reserva antioxidante medida a nivel del glóbulo rojo ${ }^{4}$.

Este desbalance entre la producción de ROS y los mecanismos de defensa antioxidantes, puede traducirse en deterioro de la función cardiaca mediada por oxidación de lípidos de membrana, ADN y diversas proteínas intracelulares, produciendo disfunción celular o muerte por apoptosis 0 necrosis $^{5}$. En efecto, estas alteraciones en el estrés oxidativo se correlacionan con parámetros funcionales, describiéndose una relación inversa entre MDA, fracción de eyección y capacidad de ejercicio ${ }^{6}$. De manera similar, en otro estudio hemos demostrado correlación entre niveles elevados de MDA con activación simpática y disminución de la respuesta cronótropa al ejercicio ${ }^{7}$.

Las ROS modulan actividades de diversas moléculas y señales de transducción intracelular en los dos principales tipos de células cardiacas: cardiomiocito y fibroblastos. En el primero, las ROS regulan proteínas involucradas en el proceso de excitación-contracción (canales de calcio en membrana celular y retículo sarcoplásmico, receptor de rianodina y miofibrillas) las que experimentan cambios en su nivel de actividad de acuerdo al estado redox intracelular ${ }^{8}$. Además, participan en mecanismos de protección miocárdica como es el precondicionamiento isquémico ${ }^{8}$. A nivel de fibroblastos, las ROS se han asociado a cambios en la producción de colágeno y fibronectina, constituyentes fundamentales de la matriz extracelular, y modificaciones en la síntesis y activación de las metaloproteasas, encargadas de la degradación y reparación de la matriz ${ }^{9,10}$.

El origen preciso de ROS en la IC es aún un tema controvertido. Las fuentes potenciales de ROS incluyen a las células inflamatorias y las mitocondrias de cardiomiocitos y fibroblastos. Los sistemas enzimáticos preponderantes en la síntesis parecen ser NADPH oxidasa y xantino oxidasa ${ }^{9}$, aun cuando pueden existir otras vías de generación de ROS en algunas etiologías particulares. A nivel miocárdico, la generación de ROS puede ser gatillada por un gran número de estímulos: isquemia y reperfusión, exposición a citoquinas infla- matorias, angiotensina II, agonistas $\alpha$-adrenérgicos, endotelina-1 y estiramiento mecánico. Todos estos estímulos convergen en la activación de la vía de $\mathrm{NF}$ kappa $\mathrm{B}^{10}$, lo que se traduce en amplificación de la respuesta inflamatoria y aumento en la síntesis de ROS mediada por NADPH oxidasa $^{11}$ y xantino oxidasa $(\mathrm{XO})^{12}$. Landmesser et al, demostraron un aumento en la actividad de XO en 14 pacientes con IC, lo que se correlacionó inversamente con la vasodilatación dependiente del endotelio ${ }^{12}$.

De manera simultánea a este aumento en el estrés redox sistémico y local, hay evidencia de disminución en los mecanismos de defensa antioxidantes, aun cuando la interpretación de estos hallazgos a nivel sistémico (actividad eritrocitaria de superóxido dismutasa (SOD), catalasa y glutatión peroxidasa (GSH-Px)) es difícil, por no reflejar necesariamente la defensa antioxidante tisular ${ }^{13}$.

Recientemente comparamos un grupo de 20 pacientes en IC con capacidad funcional II-III NYHA, estables y con terapia médica óptima con una cohorte de pacientes normales, realizando mediciones seriadas de SODec, MDA plasmático y sistemas antioxidantes eritrocitarios. En los pacientes IC, los niveles de MDA y la actividad de los sistemas antioxidantes eritrocitarios se encontraron dentro de límites normales; sin embargo, los niveles de superóxido dismutasa ligada a endotelio (SODec) mostraban una disminución significativa en relación al grupo control, sugiriendo que esta enzima podría servir como un marcador más sensible y precoz de alteraciones del status redox en pacientes en IC (Figura 2). En forma concomitante, la cohorte de pacientes en IC mostró una elevación significativa de los niveles de la proteína C ultrasensible (PCRus), lo que refleja el estado inflamatorio sistémico descrito en esta patología (Figura 3).

\section{INFLAMACIÓN EN INSUFICIENCIA CARDIACA}

Numerosas observaciones experimentales indican que los mediadores inflamatorios son importantes en la patogenia de la IC, contribuyendo al remodelado cardiaco y a las alteraciones vasculares. Diversos estudios han mostrado incrementos en los niveles plasmáticos de citoquinas inflamatorias tales como interleuquinas 1 y 6 , y factor de necrosis tumoral $\alpha$ (TNF- $\alpha)$, además de moléculas de 


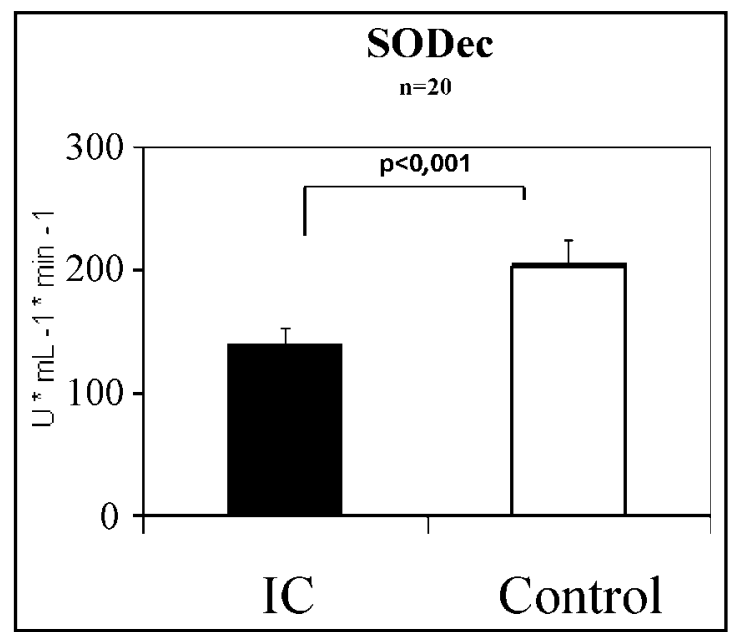

Figura 2. Niveles de superóxido dismutasa extracelular (SODec) en pacientes con insuficiencia cardiaca (IC) y controles.

adhesión (ICAM y VCAM) y niveles de $\mathrm{PCR}^{14,15}$. Esta alteración, inicialmente local, que se evidencia por niveles elevados de moléculas de adhesión en biopsias endomiocárdicas de pacientes con miocardiopatía dilatada ${ }^{16}$, es amplificada por la cascada inflamatoria mediante activación celular y expresión de neurohormonas y citoquinas proinflamatorias. En forma paralela, estas citoquinas pueden ejercer cambios en la matriz extracelular (MEC) con alteración del proceso reparativo. Estos cambios en la MEC son, al menos en parte, mediados por la activación del factor transcripcional NF-кB, que entre sus muchas acciones lleva a un aumento de la generación de ROS mediada por activación de NADPH oxidasa ${ }^{17}$. La suma de estos procesos determina fibrosis reparativa, con depósito anormal de colágeno, que reemplaza a los cardiomiocitos luego de su muerte a fin de preservar la integridad estructural del miocardio. Estos procesos alteran el remodelado ventricular y determinan finalmente disfunción ventricular ${ }^{18}$.

En modelos experimentales, altos niveles de citoquinas reproducen el fenotipo de IC, incluyendo disfunción y remodelado ventricular izquierdo, cardiomiopatía y pérdida progresiva de cardiomiocitos por apoptosis ${ }^{19}$. Observaciones en humanos ratifican este hallazgo; niveles séricos elevados de TNF- $\alpha$, interleuquina-6 y PCR se

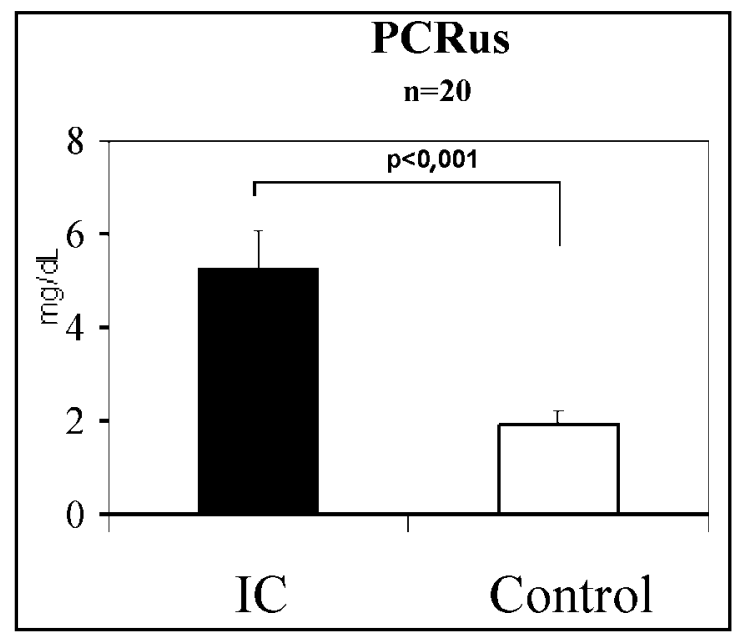

Figura 3. Niveles de proteína C ultrasensible (PCRus) en pacientes con insuficiencia cardiaca (IC) y grupo control.

asocian a un mayor riesgo de desarrollar $\mathrm{IC}^{20} \mathrm{y}$ tienen importancia pronóstica. Un ensayo clínico reciente demostró la relación entre niveles de TNF- $\alpha$ e IL-6 con una mayor mortalidad en IC ${ }^{21}$.

Además de los efectos de mediadores inflamatorios sobre la estructura y función cardiaca, existe evidencia de que ellos contribuyen a la disfunción endotelial. Prácticamente la totalidad de las condiciones de riesgo cardiovascular se asocian al desarrollo de disfunción endotelial. Los factores de riesgo cardiovascular activan una serie de genes pro-oxidantes en la pared vascular, lo que resulta en la generación de ROS que, en último término, promueven la liberación de factores de crecimiento, citoquinas, quimioatractantes y moléculas de adhesión. Esta compleja cascada de eventos promueve la transición desde la función endotelial normal hacia la disfunción endotelial, caracterizada por anomalías en el tono vasomotor, en las propiedades anticoagulantes de la superficie del endotelio, inflamación y formación de placa ateroesclerótica. Los mecanismos que median dicho cambio son múltiples. Anker et al encontraron una correlación inversa entre niveles de citoquinas inflamatorias (IL-6, TNF- $\alpha$ ) con el flujo sanguíneo de la pierna, sugiriendo que TNF- $\alpha$ puede contribuir a la debilidad y fatiga muscular en $\mathrm{IC}^{22}$. Las ROS, en particular, producen inactivación del 
óxido nítrico (NO) y reducción de su biodisponibilidad, contribuyendo a la pérdida de regulación normal del tono vascular y a cambios procoagulantes en la superficie endotelial ${ }^{23}$. En forma paralela, existe sobreexpresión de la isoforma inducible de la óxido nítrico sintetasa, correlacionada con la formación del peroxinitrito, una potente ROS. Este compuesto inhibe la acción de enzimas antioxidantes como SOD, activa metaloproteinasas y aumenta la expresión de moléculas de adhesión celular y de citoquinas inflamatorias; además, disminuye el efecto vasodilatador del $\mathrm{NO}$, lo que contribuye a la progresión de la disfunción endotelial ${ }^{24}$. Estos efectos se traducen en alteraciones clínicas objetivables mediante la pérdida de dilatación mediada por flujo a nivel de arteria braquial. Esto es concordante con nuestros hallazgos preliminares obtenidos en pacientes con IC (Tabla 1).

\section{Metaloproteasas eN LA INSUFICIENCIA CARDIACA}

El intersticio cardiaco está compuesto principalmente de fibroblastos y matriz extracelular y su función primordial es proporcionar una estructura de soporte al corazón. Poco se sabe de las alteraciones de la matriz extracelular en IC. Hay depósito excesivo de colágeno, y el colágeno III, normalmente presente (que proporciona propiedades elásticas al miocardio), es reemplazado por colágeno I, con alta fuerza tensil pero baja elasticidad. La pérdida de la estructura colágena se relaciona con falta de soporte de los cardiomiocitos, dilatación ventricular y disfunción contráctil progresiva ${ }^{19}$.

Las metaloproteasas (MMPs) son una familia de enzimas proteolíticas que degradan y remodelan la matriz extracelular, intervienen en la angio- génesis y migración celular ${ }^{25}$. En condiciones fisiológicas, su actividad es regulada por interacción con componentes específicos de la matriz, por acción de citokinas inflamatorias y ROS. Su inactivación es mediada por inhibidores endógenos tisulares llamados TIMPs ${ }^{26}$.

La participación de las MMPs y TIMPs en las enfermedades cardiovasculares comprenden los procesos de remodelado ventricular y vascular e inestabilidad de la placa aterosclerótica. La elevación de las MMPs degrada al colágeno y también promueve la pérdida de la contractilidad por proteolisis celular ${ }^{27}$. En forma similar, la pérdida de actividad de los TIMPs produce una progresiva dilatación y remodelado ventricular ${ }^{27}$. En formas experimentales y clínicas de IC, así como en la inducida por marcapaso de alta frecuencia, aumen$\tan$ las actividades de las MMPs 2 y $9^{28}$. Altieri et al encontraron niveles plasmáticos aumentados de las MMPs en 51 pacientes con IC $^{29}$. Este aumento de actividad de las MMPs puede ser mediado, al menos en parte, por ROS ${ }^{8}$. En un estudio de 47 pacientes con cardiopatía isquémica sometidos a cirugía de by-pass coronario, Kameda et al encontraron una correlación positiva entre el diámetro ventricular diastólico y las actividades de las MMP-2 y MMP-9, medidas en el líquido pericárdico lo que se correlacionó positivamente con los niveles de 8isoprostano, un marcador de estrés oxidativo ${ }^{9}$. Hallazgos similares han sido reportados por nuestro grupo en pacientes con síndromes coronarios agudos (SCA), demostrando una correlación positiva entre MDA y actividad de MMP-9, lo que sugiere un rol patogénico de ambos procesos en la remodelación que sigue al SCA. Estamos realizando investigaciones en curso que evaluarán la correlación de actividad de metaloproteinasa y de marcadores inflamatorios en pacientes con IC de etiología no isquémica. Resultados preliminares

Tabla 1. N iveles de M etaloproteinasa - 9 (M M P-9), inhibidor tisular de metaloproteinasa (T IM P-1) y función endotelial en pacientes con IC y grupo control. Los valores se muestran como promedio \pm SEM

\begin{tabular}{|lccc|}
\hline $\mathrm{n}=20$ & Controles & IC & P \\
\hline MMP-9 (normalizado MMP-2 STD) & $0,37 \pm 0,07$ & $0,39 \pm 0,10$ & 0,04 \\
TIMP-1 (veces controles) & 1 & $1,14 \pm 0,17$ & 0,22 \\
Vasodilatación dependiente de endotelio (\% cambio) & $9 \pm 3$ & $3,76 \pm 2,15$ & 0,001 \\
\hline
\end{tabular}


muestran una elevación consistente de la actividad de MMP-9 en pacientes con IC (Tabla 1).

\section{PosibILIDADES TERAPÉUTICAS EN LA INSUFICIENCIA CARDIACA}

Desde hace años es sabido que el síndrome de IC se caracteriza por activación del sistema de renina, angiotensina, aldosterona y secreción de catecolaminas y elevación de citoquinas circulantes. Esta condición, denominada activación neuroendocrina, lleva a retención progresiva de fluido y a elevación de la resistencia vascular periférica. La mayor parte de las estrategias de manejo actual en IC se basan en este paradigma. La aparición de nuevos fenómenos fisiopatológicos asociados al desarrollo de IC (inflamación, estrés redox, cambios en la matriz extracelular) y las limitaciones del tratamiento actual de la patología, han motivado una creciente investigación para evaluar los alcances terapéuticos de los hallazgos arriba reseñados. Por ejemplo, el acondicionamiento físico ha demostrado disminuir las ROS y aumentar las enzimas antioxidantes lo que se traduce en mejoría de capacidad funcional ${ }^{30}$.

A continuación describiremos algunas de las intervenciones farmacológicas orientadas a modificar estos fenómenos:

- Antioxidantes: $\mathrm{Si}$ bien existe fuerte evidencia del papel de las ROS y especies reactivas derivadas del nitrógeno, inflamación y fibrosis en la patogenia de la IC, el resultado de estudios clínicos con terapias con antioxidantes, como vitamina C, E y coenzima Q 10, no son concluyentes ${ }^{31}$. Una explicación a esto último puede ser la baja concentración de estos antioxidantes a nivel cardiaco y vascular, además de no inhibir específicamente NADPH oxidasa y xantino oxidasa sin afectar la generación de ROS a nivel cardiaco ${ }^{12}$.

- Betabloqueadores: El uso de carvedilol ha demostrado ser eficaz en disminuir el estrés oxidativo. En nuestra experiencia, el tratamiento prolongado con carvedilol se asocia a cambios favorables en la función ventricular izquierda, que se acompañan de disminución de algunos marcadores de estrés oxidativo (MDA), sin cambios en la reserva antioxidante sistémica (medida a través de SOD o GSH-Px) o en los valores de catecolaminas plasmáticas ${ }^{32}$.
- Alopurinol: Una alternativa terapéutica más específica para el manejo del estrés redox aumentado, es lo que se ha explorado mediante la utilización de alopurinol. Este fármaco actúa inhibiendo la xantino oxidasa, que constituye una importante fuente de anión superóxido en IC. En estudios clínicos el uso de alopurinol ha mostrado disminuir el consumo de oxígeno miocárdico y mejorar la función endotelial, con un perfil de seguridad conocido y a muy bajo costo ${ }^{33}$. Sin embargo, el impacto sobre endpoints clínicos no ha podido ser demostrado, persistiendo la pregunta sobre su real rol terapéutico.

- Estatinas: Son inhibidores de la 3-hidroxi-3metilglutaril-CoA reductasa con demostrada utilidad en la prevención primaria y secundaria de la enfermedad coronaria. Además de su efecto hipolipemiante, las estatinas muestran un cúmulo de acciones sobre la fisiología cardiovascular, incluyendo efectos antiinflamatorios, antitrombóticos, antiproliferativos a nivel de placa ateroesclerótica y de optimización de la función endotelial ${ }^{34}$. Son estas propiedades, denominadas habitualmente 〈efectos pleiotrópicos de las estatinas», las que las perfilan como una potencial alternativa terapéutica en IC. En estudios prospectivos en IC, la hipocolesterolemia ha demostrado una asociación con mayor mortalidad ${ }^{34}$, lo que a priori permitiría anticipar un efecto deletéreo de fármacos hipolipemiantes en la historia natural de la IC. Sin embargo, evidencia preliminar parece mostrar lo contrario. El análisis retrospectivo de una cohorte de 840 pacientes en IC demostró una asociación entre el uso de estatinas y mortalidad cardiovascular disminuida independiente de la etiología de la $\mathrm{IC}^{35}$. Estos hallazgos han sido ratificados por otros estudios observacionales. Recientemente se ha reportado que el uso de atorvastatina en IC ha mostrado disminuir los niveles de PCR, TNF- $\alpha$ e IL-636; además, se ha descrito mejoría en función ventricular y capacidad de ejercicio en terapias más prolongadas ${ }^{37}$. Los efectos beneficiosos de las estatinas en IC pueden depender, al menos en parte, de su habilidad para inhibir la formación de proteínas llamadas isoprenoides en las paredes vasculares, evitando la activación de proteínas $\mathrm{G}$ pequeñas (Rho y Ras), lo que a su vez inhabilita las vías de inflamación. De manera similar, parecen optimizar la función endotelial mediante el 
aumento en la producción de NO y disminución en los niveles de endotelina $-1^{34}$.

Actualmente, nuestro grupo realiza estudios que busca esclarecer el potencial rol terapéutico de estatinas y alopurinol en IC. Además, existen estudios en curso que pretenden evaluar el impacto de terapias prolongadas con estatinas en mortalidad y eventos cardiovasculares mayores en el seguimiento a largo plazo de pacientes con $\mathrm{IC}^{38}$.

Otros blancos terapéuticos potenciales están orientados a aumentar la reserva antioxidante sistémica y tisular mediante el uso de inhibidores

\section{REFERENCIAS}

1. Grieve DJ, ShaH AM. Oxidative stress in heart failure. More than just damage. Eur Heart J 2003; 24: 2161-216.

2. Pacher $P$, Schulz R, Laudet L, Szabo C. Nitrosative stress and pharmacological modulation of heart failure. TRENDS Pharmacol Sciences 2005; 26: 165-76.

3. Kenneth E, López J, Buckier K, Archer S. Acute Oxygen-Sensing Mechanisms. N Engl J Med 2005; 353: 2042-55.

4. Castro P, Díaz G, Nettle D, Corbalán R, Pérez O, Lavandero $S$ et aL. Effects of Early Decrease in Oxidative Stress After Medical Therapy in Patients With Class IV Congestive Heart Failure. Am J Cardiol 2002; 89: 243-54.

5. González A, Fortuno MA, Querejeta R, Ravassa S, LóPEz B, LóPez N. Cardiomyocyte apoptosis in hypertensive cardiomyopathy. Cardiovasc Res 2003; 59: 549-62.

6. Flora SAM, David L, Pimental R, Mulukutia S, Tabaee A, BRIStow R ET AL. Increased Reactive Oxygen Species Production and Functional Alterations in Antioxidant Enzymes in Human Failing Myocardium. Congest Heart Fail 2005; 11: 2130-44.

7. Castro P, Greig D, Pérez O, Chiong M, Díaz G, VuKasOVIC JL ET AL. Relation Between Oxidative Stress, Catecholamines, and Impaired Chronotropic Response to Exencise in Patients With Chronic Heart Failure Secondary to Ischemic or Idiopathic Dilated Cardiomyopathy. Am J Cardiol 2003; 92: 215-8.

8. Poli G, Leonarduzzi G, Biasi F, Chiarpotto E. Oxidative stress and cell signalling. Curr Med Chem 2004; 11: 1163-82. de NADPH oxidasa y de peroxinitrito, los que han mostrado efectos beneficiosos en modelos anima$\operatorname{les}^{39}$. De manera similar, pequeños ensayos clínicos con inhibidores de las MMPs (doxiciclina) se han asociado a disminución de marcadores inflamatorios en pacientes posinfarto 40 .

En suma, en la IC existen fenómenos de estrés oxidativo, inflamación y disfunción endotelial que se interrelacionan en forma compleja y que pueden ser revertidos por intervenciones terapéuticas. El real impacto sobre las manifestaciones clínicas y progresión de la IC está por definirse.

9. Kameda K, Matsunaga T, Abe N, Hanada H. Correlation of oxidative stress with activity of matrix metalloproteinase in patients with coronary artery disease. Possible role for left ventricular remodelling. Eur Heart J 2003; 24: 2180-5.

10. FinKel T. Signal transduction by oxidative reactive species in non-phagocytic cells. J Leukocyte Biol 1999; 65: 337-40.

11. Griendung KK, Sorescu D, Ushio M. NAD(P)H oxidase: role in cardiovascular biology and disease. Circ Res 2000; 86: 494-501.

12. Landmesser U, Siekermann S, Dikalov S, Tatge H, WiLKe R, Kohler C et al. Vascular oxidative stress and endothelial dysfunction in patients with chronic heart failure: role of xanthine-oxidase and extracellular superoxide dismutase. Circulation 2002; 106: 3073-8.

13. Pérez O, Castro P, Díaz G, Nettle D, Moraga F, Chiong M et al. Persistencia del estrés oxidativo postrasplante cardiaco: estudio comparativo entre pacientes con trasplante cardiaco y con insuficiencia cardiaca crónica estable. Rev Esp Cardiol 2002; 55: 831-7.

14. Paulus WJ. Cytokines and heart failure. Heart Fail Monit 2000; 1: 50-6.

15. ABrams J. C-reactive protein, inflammation, and coronary risk: an update. Cardiol Clin 2003; 21: 327-31.

16. Noutsias M, SeeBerg B, Schultheiss H-P. Expression of cell adhesion molecules in dilated cardiomyopathy. Circulation 1999; 99: 2124-31.

17. Ammar FZ, Gannon PO, Amiri F, Schiffrin E. Fibrosis, matrix metalloproteinases, and inflammation in the heart of DOCA-salt hypertensive rats: role of ETA receptors. Hypertension 2002; 39: 679-84. 
18. Jalil JE, Doering CW, Janicki JS, Pick R, Shroff SG, WEBER KT. Fibrillar collagen and myocardial stiffness in the intact hypertrophied rat left ventricle. Circ Res 1999; 64: 1041-50.

19. Thaik CM, Calderone A, TaKahashi N, Colucci WS. Interleukin-1 modulates the growth and phenotype of neonatal rat cardiac myocytes. J Clin Invest 1995; 96: 1093-9.

20. Satoh S, Oyama J, Suematsu N, Kadokami T. Increased productivity of tumor necrosis factor-alpha in helper $\mathrm{T}$ cells in patients with systolic heart failure. Inter J Cardiol 2005; 26: 1298-304.

21. Desswal A, Petersen NJ, Feidman AM, Young JB, White BB, ManN DL. Cytokines and cytokine receptors in advanced heart failure: an analysis of the cytokine database from the Vesnarinone trial (VEST). Circulation 2001; 103: 2055-9.

22. AnKer SD, Volterrani M, Egerer KR, Felton CV, Kox WJ, Poole PA, Coats AJ. Tumor necrosis factor as a predictor of impaired peak leg blood flow in patients with chronic heart failure. QJM 1998; 91: 199-203.

23. Sharma $R$, Davidoff MN. Oxidative stress and endothelial dysfunction in heart failure. Congest Heart Fail 2002; 8: 165-72.

24. LALu MM. Peroxynitrite in myocardial ischemiareperfusion injury. Heart Fail Rev 2002; 7: 359-69.

25. VisSE R, NAGASE H. Matrix metalloproteinases and tissue inhibitors of metalloproteinases. Structure, function, and biochemistry. Circ Res 2003; 92: 827-39.

26. SteRNLCHT MDL, WeRB Z. How matrix metalloproteinases regulate cell behavior. Annu Rev Cell Dev Biol 2001; 17: 463-516.

27. Spinale FG, Coker ML, Heung LJ, Bond BR. A matrix metalloproteinase induction/activation system exists in the human left ventricular myocardium and is upregulated in heart failure. Circulation 2000; 102: 1944-9.

28. Tsuruda T, Costello LC, Burnett JC. Matrix metalloproteinases: pathways of induction by bioactive molecules. Heart Fail Rev 2004; 9: 53-61.

29. Altieri P, Brunew S, Garibaidi S, Nicolino A, Ubaldi S, Spallarossa L et al. Metalloproteinases 2 and 9 are increased in plasma of patients with heart failure. Eur J Clin Invest 2003; 33: 648-56.

30. Linke A, Adams V, Christian P, ERbs S, Gielen S. Antioxidative Effects of Exercise Training in Patients With Chronic Heart Failure: Increase in
Radical Scavenger Enzyme Activity in Skeletal Muscle. Circulation 2005; 111: 1763-70.

31. BERRY CE, HERE JM. Xanthine oxidoreductase and cardiovascular disease: molecular mechanisms and pathophysiological implications. J Physiol 2004; 555: 589-606.

32. Castro P, Vukasovic Jl, Chiong M, díaz G, Alcaíno $H$, Copaja $M$ ET aL. Effects of carvedilol on oxidative stress and chronotropic response to exercise in patients with chronic heart failure. Eur J Heart Fail 2005; 7: 1033-9.

33. Gavin AD, Struthers AD. Allopurinol reduces Btype natriuretic peptide concentrations and haemoglobin and impairs ventricular function but does not alter exercise capacity in chronic heart failure. Heart 2005; 91: 749-53.

34. NaKagami H, Jensen KS, Lao JK. A novel pleiotropic effect of statins: prevention of cardiac hypertrophy by cholesterol-independent mechanisms. Ann Med 2003; 35: 398-403.

35. Folkeringa RJ, Van Kraaij DJ, Tiejeman RG, Nieman FH, PINTO YM, CRJjNS HJ. Statins associated with reduced mortality in patients admitted for congestive heart failure. J Card Fail 2006; 12: 134-8.

36. Wojnicz R, Wilczek K, Nowalany E, Szygula B, NowAK J ET AL. Usefulness of atorvastatin in patients with heart failure due to inflammatory dilated cardiomyopathy and elevated cholesterol levels. Am J Cardiol 2006; 97: 899-904.

37. Srikanth S, Muhammad Q, Stamatios L, Neeraj T. Atorvastatin Improves Left Ventricular Systolic Function and Serum Markers of Inflammation in Nonischemic Heart Failure. JACC 2006; 47: 332-7.

38. Tavazzi L, Tognoni G, Franzosi Mg, Latini $R$, Maggioni Ap, Marchiol R. Rationale and design of the GISSI heart failure trial: a large trial to assess the effects of $n-3$ polyunsaturated fatty acids and rosuvastatin in symptomatic congestive heart failure. Eur J Heart Fail 2004; 6: 531-3.

39. Pacher P, Morris O, Claude R. Role of nitrosative stress and peroxynitrite in the pathogenesis of diabetic complications. Emerging new therapeutical strategies. Curr Med Chem 2005; 12: 267-75.

40. Campt TM, Braun JC, Powak D, Gavin L. Doxycycline ameliorates ischemic and borderzone remodeling and endothelial dysfunction after myocardial infarction in rats. J Heart Lung Transplant 2004; 23: 729-36. 